\title{
Neural Variability Is Quenched by Attention
}

\author{
(D)Ayelet Arazi, ${ }^{1,2}$ Yaffa Yeshurun, ${ }^{3}$ and Ilan Dinstein ${ }^{1,2,4}$ \\ ${ }^{1}$ Department of Brain and Cognitive Sciences, ${ }^{2}$ Zlotowski Center for Neuroscience, Ben Gurion University of the Negev, Beer-Sheva, 8410501, Israel, \\ ${ }^{3}$ Department of Psychology, University of Haifa, Haifa, 3498838, Israel, and ${ }^{4}$ Department of Psychology, Ben Gurion University of the Negev, Beer-Sheva, \\ 8410501, Israel
}

Attention can be subdivided into several components, including alertness and spatial attention. It is believed that the behavioral benefits of attention, such as increased accuracy and faster reaction times, are generated by an increase in neural activity and a decrease in neural variability, which enhance the signal-to-noise ratio of task-relevant neural populations. However, empirical evidence regarding attention-related changes in neural variability in humans is extremely rare. Here we used EEG to demonstrate that trial-by-trial neural variability was reduced by visual cues that modulated alertness and spatial attention. Reductions in neural variability were specific to the visual system and larger in the contralateral hemisphere of the attended visual field. Subjects with higher initial levels of neural variability and larger decreases in variability exhibited greater behavioral benefits from attentional cues. These findings demonstrate that both alertness and spatial attention modulate neural variability and highlight the importance of reducing/quenching neural variability for attaining the behavioral benefits of attention.

Key words: alertness; neural variability; spatial attention; variability quenching

\section{Significance Statement}

Attention is thought to improve perception by increasing the signal-to-noise ratio of the neuronal populations that encode the attended stimulus. Signal-to-noise ratio can be enhanced by increasing neural response (signal) and/or by reducing neural variability (noise). The ability of attention to increase neural responses has been studied extensively, but the effects of attention on neural variability have rarely been examined in humans. Here, we demonstrate that modulating different components of attention, including alertness and spatial attention, reduces neural variability in humans. Furthermore, we show that subjects with larger reductions in neural variability exhibit greater behavioral benefits from attention. These results demonstrate that reduction of neural variability is a fundamental feature of attentional processes in humans with clear behavioral importance.

\section{Introduction}

Neural activity is variable over time and across trials, as demonstrated by numerous studies of the mammalian brain (Arieli et al., 1996; Carandini, 2004; Churchland et al., 2010; Goris et al., 2014). The variability of neural responses across trials containing identical stimuli is large before stimulus onset and significantly reduced ("quenched") following stimulus presentation. This trial-by-trial variability quenching is a general cortical phenomenon, which is apparent in multiple different brain areas, when using different types of stimuli and tasks, as measured with electrophysiology in animals (Monier et al., 2003; Finn et al., 2007;

Received Feb. 12, 2019; revised April 8, 2019; accepted April 21, 2019.

Author contributions: A.A., Y.Y., and I.D. designed research; A.A. performed research; A.A. analyzed data; A.A. wrote the first draft of the paper; A.A., Y.Y., and I.D. edited the paper; A.A., Y.Y., and I.D. wrote the paper.

This work was supported by Israel Science Foundation Grant 961/14 to I.D. and Israel Academy of Sciences Adams Fellowship to A.A.

The authors declare no competing financial interests.

Correspondence should be addressed to Ayelet Arazi at araziay@post.bgu.ac.il.

https://doi.org/10.1523/JNEUROSCI.0355-19.2019

Copyright $\odot 2019$ the authors
Churchland et al., 2010; Hussar and Pasternak, 2010) or using noninvasive neuroimaging techniques in humans (Schurger et al., 2010, 2015; He, 2013; Arazi et al., 2017a). In this study, we quantified the effects of attention on neural variability and variability quenching.

Attention is commonly viewed as an "umbrella term" that is comprised of several components, including alertness (i.e., arousal or vigilance) and spatial orienting (i.e., spatial attention) (Petersen and Posner, 2012). Alertness is often studied by presenting cues that inform participants that something is about to happen, regardless of where it will happen. Such cues improve the reaction times and accuracy rates of subjects on a variety of tasks (Fan et al., 2002, 2009). Spatial orienting requires information about the spatial location of an upcoming task-related stimulus. Allocating spatial attention improves the speed and accuracy of behavioral responses to stimuli at the attended location, at the expense of stimuli located elsewhere (Yeshurun and Carrasco, 1998; Carrasco, 2011). The neural mechanisms underlying these behavioral effects have been a topic of extensive research. 
Electrophysiological studies have shown that modulating alertness (Bromberg-Martin et al., 2010a,b) or spatial attention (Mitchell et al., 2007; Buffalo et al., 2010; Thiele et al., 2016) increases the mean firing rate of single neurons encoding the attended stimulus, across multiple stages of visual processing. Similarly, fMRI studies in humans have reported that modulating alertness (Fan et al., 2005; Xuan et al., 2016) or spatial attention (Gandhi et al., 1999; Kastner et al., 1999; Silver et al., 2007; Saproo and Serences, 2010) increases BOLD responses in the relevant areas of visual cortex. In addition to increased firing rates, studies in nonhuman primates have demonstrated that spatial attention also reduces firing rate variability in individual neurons as measured by the Fano factor (ratio between the variance of firing rate and its mean) (Mitchell et al., 2007; Thiele et al., 2016). Furthermore, spatial attention reduces the correlated variability across pairs of neurons in local populations of visual cortex neurons (Cohen and Maunsell, 2009; Mitchell et al., 2009). Increased firing rates and decreased variability improve the neural signal-tonoise ratio, which may explain why modulating attention can improve perception of the attended stimulus (Ress et al., 2000; Noudoost et al., 2010; Moore and Zirnsak, 2017). While signal enhancement has been studied extensively in both humans and nonhuman primates, changes in neural variability as a result of spatial attention modulations have never been studied in humans, and only one study has examined changes in neural variability as a function of alertness (Broday-Dvir et al., 2018).

To address these issues, we measured trial-by-trial variability with EEG in two separate experiments. In one experiment, a stimulus was briefly presented in the right or left visual field and subjects reported its orientation. Spatial attention was manipulated by presenting a cue that was mostly informative regarding the location of an upcoming stimulus (Posner, 1980) (Fig. 1A). In a second control experiment, subjects passively observed identical cues without a task (i.e., the cues were meaningless). We compared neural variability across the two experiments and across conditions where attention was allocated to the left or right visual fields. This allowed us to quantify changes in cortical neural variability as a function of task demands (i.e., attended vs passive; probing the effects of alertness) and spatial attention (i.e., attend right vs left; probing the effects of spatial orienting).

\section{Materials and Methods}

Subjects

Twenty-seven subjects ( 16 females, mean age: $24.6 \pm 1.5$ years) participated in the study. All subjects had normal or corrected-to-normal vision. The Ben-Gurion University Internal Review Board approved the study. Subjects provided written informed consent and were paid for their participation or received research credit.

\section{Experimental design}

The experiment was performed in a dark and soundproof room. The stimuli were presented using MATLAB (The MathWorks) and Psychtoolbox (Brainard, 1997).

Stimuli. A white fixation cross and two white square frames $\left(4.3^{\circ} \times\right.$ $4.3^{\circ}$ ) were continuously displayed on a gray background throughout the experiment. The fixation cross was located at the center of the screen, and the centers of the white frames were located $11.1^{\circ}$ to the left/right of the fixation cross. Each trial began with the presentation of a cue consisting of a white arrow $\left(1.69^{\circ} \times 0.6^{\circ}\right)$ that appeared in the center of the screen and pointed to the left, to the right, or to both sides (double-headed arrow). The cue appeared for $100 \mathrm{~ms}$ and was followed by a cue-target interval of $500 \mathrm{~ms}$. The target stimulus consisted of a circle with black and white stripes and a diameter of $2.98^{\circ}$. The target stimulus appeared in the center of the left or right frame for $50 \mathrm{~ms}$ (Fig. 1A).

Orientation discrimination task. Subjects were instructed to report whether the target stimulus was tilted to the right or left of vertical (tilt
A

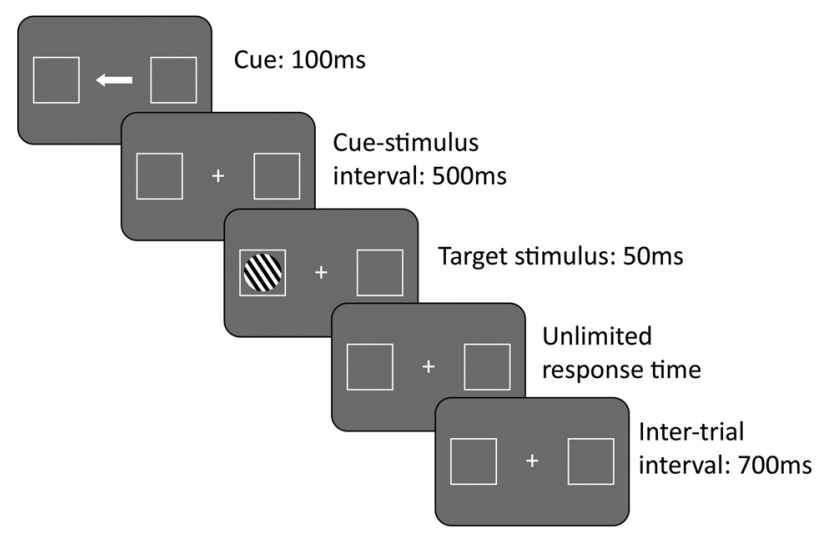

B
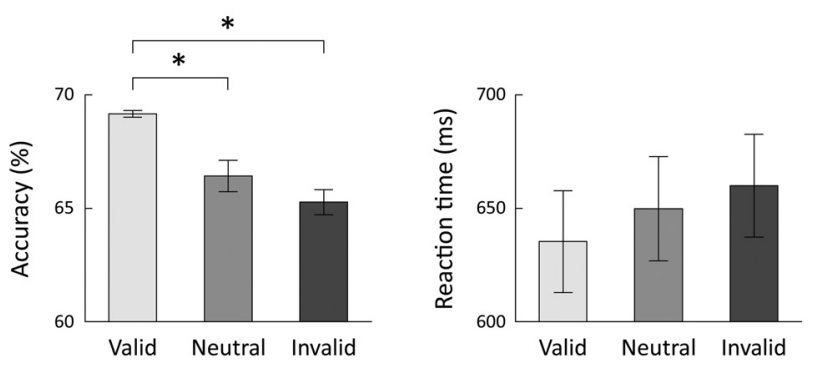

Figure 1. Experimental design and behavioral results. $\boldsymbol{A}$, Experimental design of the orientation discrimination task. Each trial began with the presentation of a central cue (arrow) presented for $100 \mathrm{~ms}$, which pointed to the left, right, or both sides. The cue was followed by a target stimulus, which consisted of black-and-white stripes and presented for $50 \mathrm{~ms}$ in the right or left visual field. Subjects reported whether the target stimulus was tilted to the left or right of vertical. The cue accurately predicted the target location in $60 \%$ of the trials (valid), pointed to the opposite location in $20 \%$ of the trials (invalid), and pointed to both locations in $20 \%$ of the trials (neutral). $\boldsymbol{B}$, Mean accuracy rates (left) and reaction times (right) across subjects for each of the three conditions (valid, invalid, and neutral). Error bars indicate SEM across subjects. ${ }^{*} p<0.01$, significant differences across conditions (post hoc Tukey's test).

order was randomized) by pressing two different keys. Auditory feedback ( $1000 \mathrm{~Hz}$ pure tone; stimulus duration: $50 \mathrm{~ms}$; stimulus intensity: $55 \mathrm{db}$ ) was given after correct responses only, followed by an intertrial interval of $700 \mathrm{~ms}$ (Fig. 1A). The cue accurately pointed to the location of the target stimulus in $60 \%$ of the trials (valid condition), pointed to the opposite direction in $20 \%$ of the trials (invalid condition), and pointed both ways in $20 \%$ of the trials (neutral condition). Subjects completed 10 blocks of 120 trials each, for a total of 1200 trials (720 valid trials, 240 invalid trials, and 240 neutral trials).

The orientation angle of the target stimulus was controlled throughout the experiment using a 2-down-1-up staircase procedure, which was applied to the valid trials only. The initial angle on each block was $4^{\circ}$. The angle was divided by a factor of $\sqrt{2}$ after two correct responses or multiplied by a factor of $\sqrt{2}$ after one incorrect response, thereby maintaining an accuracy rate of $\sim 70 \%$ in the valid trials. This procedure was used to avoid ceiling or floor effects.

Control experiment. In this version of the experiment, the fixation cross, frames, and cues were presented as described above, but the orientation discrimination task was omitted. The target was not presented and subjects did not respond (i.e., trails ended $700 \mathrm{~ms}$ after cue presentation). Subjects were instructed to maintain fixation and passively observe three blocks of 300 trials each. Subjects performed this experiment before the orientation discrimination task to avoid explicit manipulation of their spatial attention.

\section{Behavioral data analysis}

Mean accuracy rates and mean reaction times were computed for each subject and for each attentional condition in the orientation discrimina- 
tion task (i.e., valid, invalid, and neutral). The first 20 trials in each block and trials with reaction time $<200 \mathrm{~ms}$ or $>1500 \mathrm{~ms}$ were excluded from the analysis. Trials with incorrect responses were excluded from the reaction time analysis. The overall perceptual performance of individual subjects was quantified by calculating the mean tilt angle of the target stimulus across all valid trails (smaller angles = better perceptual performance). The benefit of attention ("attentional effect") was calculated for each subject as the difference between the mean accuracy rate in the valid and invalid conditions.

\section{EEG and eye tracking recordings}

EEG data were recorded using a 64-channel BioSemi ActiveTwo system connected to a standard EEG cap according to the international 10-20 system. EEG data were sampled at a rate of $1024 \mathrm{~Hz}$ and referenced to the vertex electrodes. Electrode offsets were kept between $\pm 40 \mathrm{mV}$ as recommended by the BioSemi operating guideline (www.biosemi.com). EOG was recorded using two electrodes at the outer canthi of the left and right eyes and one electrode placed below the right eye. The position of the left eye was recorded simultaneously using an eye tracker (EyeLink 1000; SR Research) at a sampling rate of $1000 \mathrm{~Hz}$.

\section{EEG preprocessing}

Data were analyzed using MATLAB and EEGLAB toolbox (Delorme and Makeig, 2004). Data were rereferenced to the bilateral mastoid electrodes, downsampled to $512 \mathrm{~Hz}$, and filtered using 1-40 Hz bandpass filter. We extracted trials containing the responses to the cue, for each cue type, in a time window starting $200 \mathrm{~ms}$ before cue onset and ending 500 $\mathrm{ms}$ after cue onset. Baseline correction (i.e., subtracting the mean of the precue period from each trial) was not performed to preserve trial-bytrial variability in the precue period.

Trials containing absolute amplitudes that exceeded $70 \mu \mathrm{V}$ in frontal electrodes or power that exceeded $25 \mathrm{db}$ in the $20-40 \mathrm{~Hz}$ frequency band were identified as containing eye blinks or muscle artifact, respectively, and removed from the analysis. Identification of eye blinks was confirmed by eye tracking. In addition, trials with horizontal or vertical eye movement that exceeded 100 pixels from the fixation cross (equivalent to $2.5^{\circ}$ visual angle) were identified as trials with saccades and removed from further analysis. In the orientation discrimination experiment, trials with incorrect response were excluded from the EEG analyses. Mean number of trials across subjects after trial rejection in the control experiment was $205(\mathrm{SD}=48)$ for cue right, $206(\mathrm{SD}=43)$ for cue left, and 199 $(\mathrm{SD}=43)$ for double-headed cue. Mean numbers of trials in the discrimination experiment was $270(S D=38)$ for cue right, $270(S D=37)$ for cue left, and $134(\mathrm{SD}=21)$ for double-headed cue.

\section{EEG data analysis}

Trial-by-trial variability. We analyzed trials of each cue type separately (i.e., left, right, and double-headed arrow). Trial-by-trial neural variability was computed for each subject in each of the 64 electrodes separately. We computed the variance across trials for each time point in the extracted epochs ( $-200 \mathrm{~ms}$ precue to $500 \mathrm{~ms}$ postcue). We also calculated a single measure of precue variability by computing the mean trial-bytrial variability across time points from $-200 \mathrm{~ms}$ to cue onset. An equivalent measure of postcue variability was computed as the mean variability across time points from 250 to $500 \mathrm{~ms}$ after presentation of the cue.

Electrodes of interest. We focused our analyses on eight parietal electrodes $(\mathrm{CP} 1, \mathrm{CP} 2, \mathrm{P} 1, \mathrm{P} 2, \mathrm{P} 3, \mathrm{P} 4, \mathrm{PO} 3$, and $\mathrm{PO} 4)$ that exhibited the largest responses to the cue and visual stimulus.

Lateralization index. To examine the spatial topography of trial-bytrial variability as a function of cued/attended visual field, we subtracted the postcue variability of the trials where the cue pointed to the right from the postcue variability of the trials where the cue pointed to the left, and divided by the mean of variability across both cue types as follows: Var $_{\text {right }}-$ Var $_{\text {left }}$ $\frac{V a r_{\text {right }}-V a r_{\text {left }}}{\text { mean }\left(\operatorname{Var}_{\text {right }}, \operatorname{Var}_{\text {left }}\right)}$. This was performed for each electrode separately, enabling us to map differences across electrodes.

\section{Statistical analysis}

Comparisons across behavioral measures were performed using a oneway ANOVA with the attentional condition as the only factor, followed by post hoc Tukey's tests when the results indicated significant differences. Comparisons across experiments were performed using two-tailed paired-sample $t$ tests. We examined the relationships between behavioral and EEG measures using Pearson's correlation. The statistical significance of the correlation coefficients was assessed with a randomization test where we shuffled the labels of the subjects before computing the correlation coefficient. We performed this procedure 10,000 times while shuffling the labels across subjects randomly each time to generate a null distribution for each pair of EEG/behavioral measures. For the true correlation coefficient to be considered significant, it had to be higher than the 97.5th percentile or lower than the 2.5th percentile of the null distribution (equivalent to $p=0.05$ in a two-tailed $t$ test).

\section{Electrodes offset and gaze variability}

To assess the quality of individual EEG recordings, we examined the electrode offset measure, which is commonly used in studies using EEG systems with active electrodes, where there is no direct measurement of impedance (Kappenman and Luck, 2010). For each subject, we calculated both the mean electrode offset and its trial-by-trial variability. The mean offset value was first calculated for each trial (from $-200 \mathrm{~ms}$ precue to $500 \mathrm{~ms}$ postcue), and we then computed the mean or variability across trials for each experiment.

We also examined the mean gaze position and its variability across trials in each of the subjects. We computed the Euclidean distance of gaze position from the fixation cross for each time point in the experiment. The Euclidean distance was computed as the square root of the sum of squares of the horizontal and vertical gaze position relative to the fixation cross in units of visual angle. This enabled us to measure the mean gaze position and its SD across trials for each time point from $-200 \mathrm{~ms}$ precue to $500 \mathrm{~ms}$ postcue. We also calculated a single measure of gaze variability for each subject by computing the mean gaze variability in the postcue period (from 0 to $500 \mathrm{~ms}$ ) separately for each experiment and cue type.

\section{Results}

There were significant differences in the subjects' accuracy rates across conditions in the orientation discrimination experiment (one-way ANOVA, $F_{(2,78)}=14.85, p<0.001$ ). Subjects performed the task with higher accuracy rates in valid trials compared with both neutral and invalid trials (post hoc Tukey's tests, $p<0.01)$. There was a trend for faster reaction times in the valid trials versus the neutral and invalid trials, but these differences did not reach statistical significance (Fig. $1 B$ ). These results demonstrate that subjects were attending the cues/arrows and that the cue was beneficial for accurately performing the task in the valid trials, where the arrow correctly indicated the location of the upcoming stimulus.

\section{Alertness reduces trial-by-trial neural variability}

In the control experiment, identical cues were presented without a task, making them meaningless. There was, therefore, no motivation for subjects to attend or change their alertness in response to the cues. Comparing neural variability in the control experiment with that in the orientation discrimination experiment allowed us to examine the effects of general attention and alertness on neural variability. Trial-by-trial variability was reduced (i.e., quenched) $\sim 200 \mathrm{~ms}$ after the presentation of the cues in both the control and orientation discrimination experiments (Fig. 2). Variability quenching was larger for all cue types in the discrimination experiment than in the control experiment in both right and left occipital electrodes (Fig. 2A). These differences in neural variability across the experiments were specific to the occipital electrodes (Fig. $2 B$ ), suggesting that general attention and alertness to the task in the discrimination experiment were associated with a selective decrease in the variability of visual neural activity across trials. 
A

\section{Right electrodes}
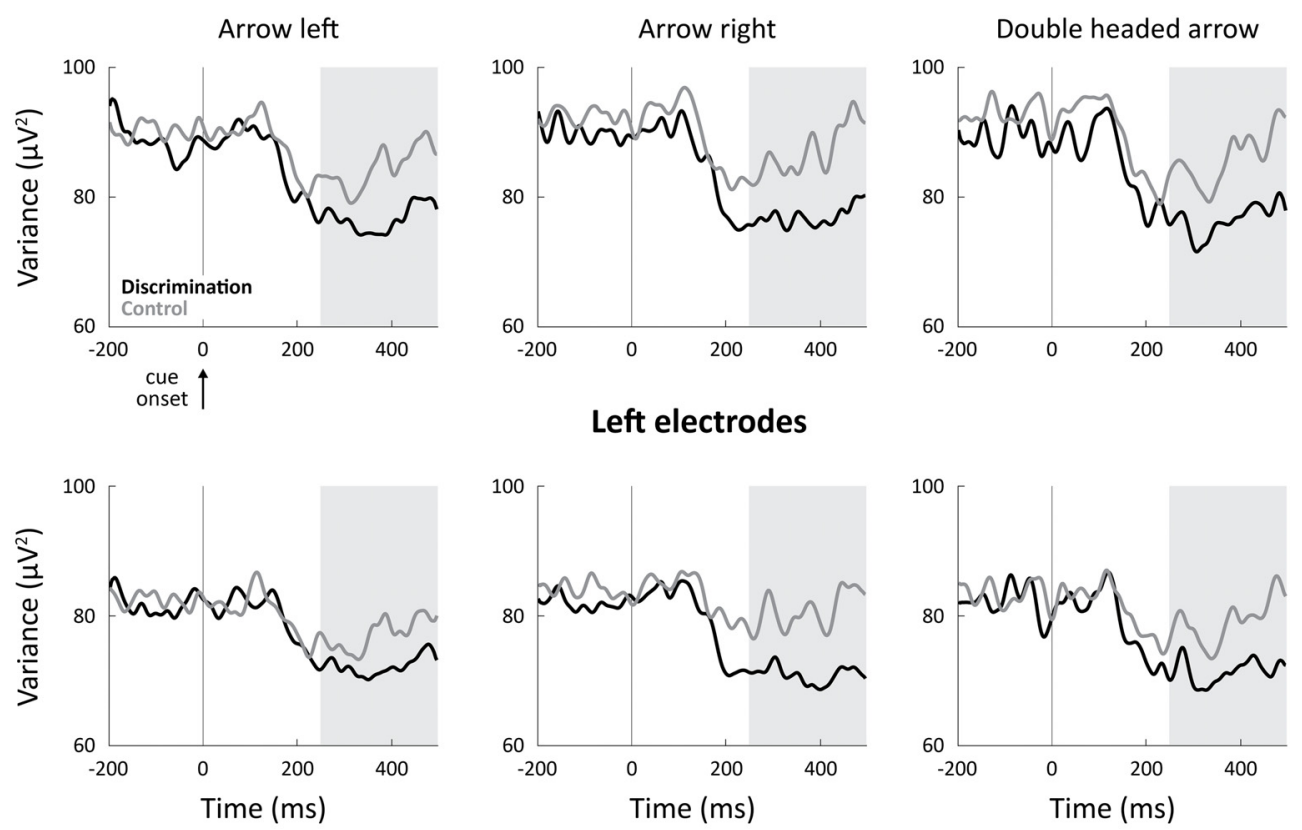

B

Differences in trial-by-trial variability between control and discrimination experiments

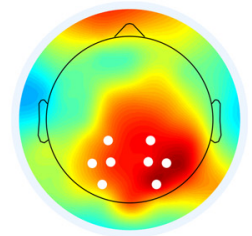

Arrow left

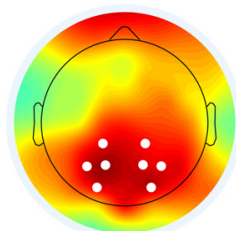

Arrow right
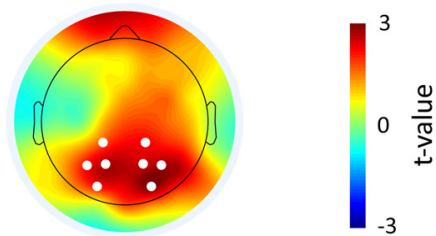

Double headed arrow

Figure 2. Alerting subjects to an upcoming task-relevant stimulus reduced trial-by-trial variability in occipital and parietal cortices. $A$, Temporal dynamics of trial-by-trial variability during the control (gray) and discrimination (black) experiments. Each panel represents the results of a different cue type (left, right, and double-headed) in the right (top panels) and left (bottom panels) electrodes. Gray background represents $250-500$ ms time window with sustained variability quenching. Vertical line indicates cue onset. $\boldsymbol{B}$, Spatial topography of variability differences between the control and orientation discrimination experiments. A map of $t$ values demonstrates the topography of electrodes with significant differences in neural variability between the experiments, $250-500 \mathrm{~ms}$ after the cue presentation. White dots represent electrodes that were selected for the variability analyses.

We quantified the neural variability magnitude in the postcue window (250-500 ms; Fig. 2A, marked in gray) for each cue type in each of the experiments. Variability in this time window was significantly smaller in the discrimination experiment compared with the control experiment for all cue types in both right and left electrodes (two-tailed $t$ test, $p<0.05$; Fig. 3), except for the left arrow cue in the left electrodes (the trend in this case was also in the correct direction). In contrast, there were no significant differences in neural variability across experiments in the precue interval (from $-200 \mathrm{~ms}$ to cue onset; two-tailed $t$ tests, $p>0.46$ ). Together, these results demonstrate that alerting the subjects to an upcoming task-relevant stimulus caused a reduction in trialby-trial neural variability (i.e., increased variability quenching).

\section{Spatial attention reduces trial-by-trial variability}

Comparing trial-by-trial variability as a function of cue type in the orientation discrimination experiment allowed us to examine the effects of spatial attention. Allocating spatial attention to one
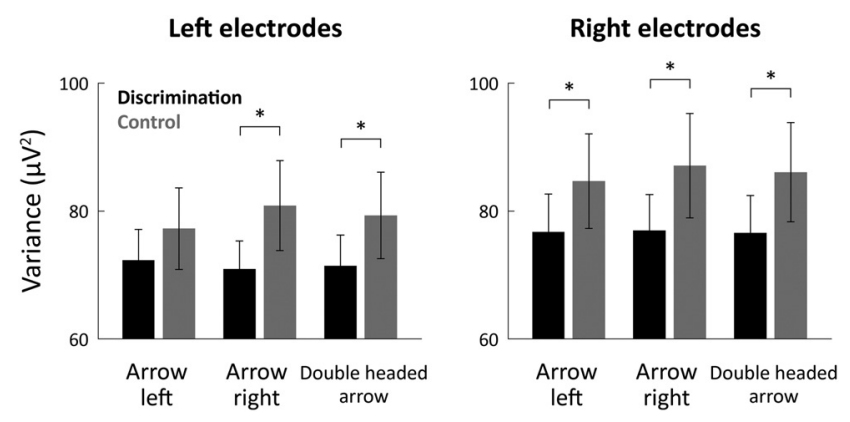

Figure 3. Neural variability was significantly smaller in the orientation discrimination experiment. Mean trial-by-trial variability across subjects during the postcue time window $(250-500 \mathrm{~ms})$. Results are presented for each cue type (left, right, and doubleheaded) in the control (gray bars) and orientation discrimination (black bars) experiments. Neural variability was measured separately in the left (left panel) and right (right panel) electrodes. Error bars indicate SEM across subjects. ${ }^{*} p<0.05$, significant differences across experiments (two-tailed $t$ test). 


\section{Discrimination experiment}

A

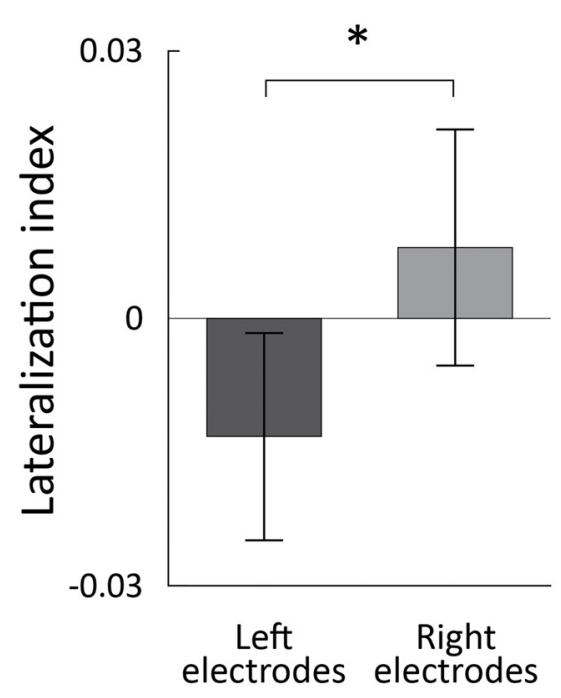

B

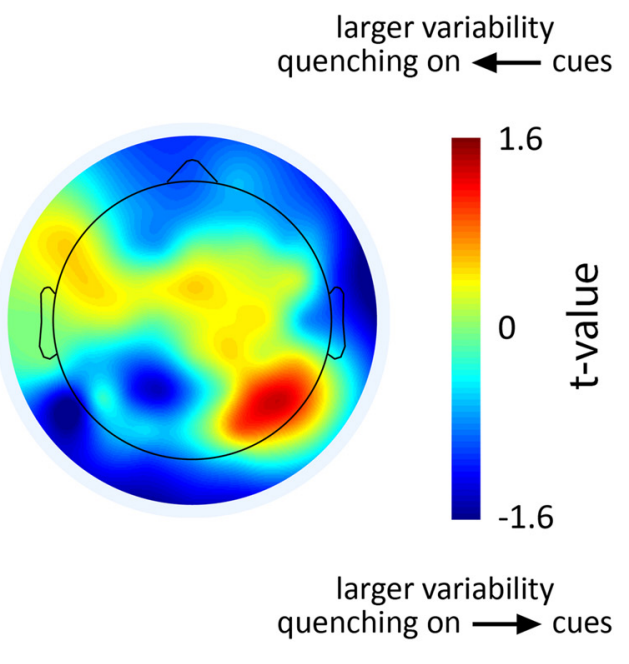

\section{Control experiment}

C

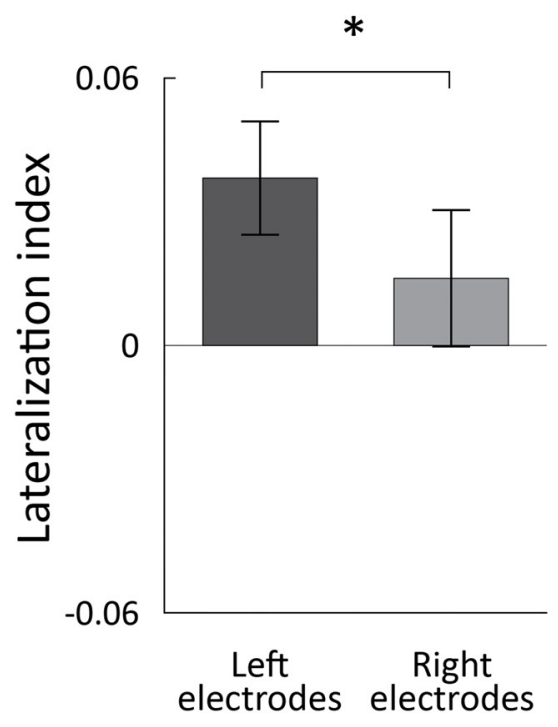

D

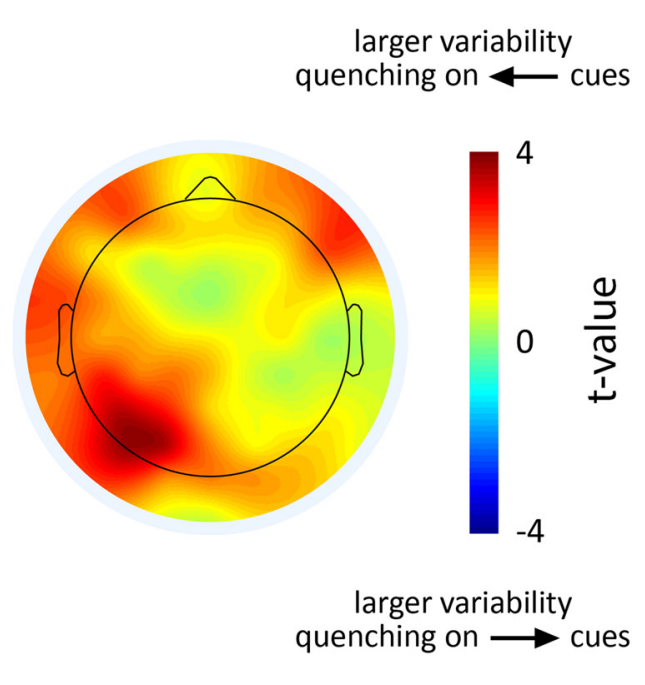

Figure 4. Variability quenching during the discrimination, but not the control experiment, was larger in the contralateral hemisphere to the attended visual field. $\boldsymbol{A}$, Mean lateralization index across the four selected electrodes in the right and left hemispheres, respectively, in the discrimination experiment. Error bars indicate SEM across subjects. $\boldsymbol{B}$, Spatial topography of lateralization indexes per electrode in the discrimination experiment. We computed the lateralization index for each electrode in each subject and then performed a $t$ test, across subjects, to determine whether indexes were different from zero. Positive $t$ values in the right electrodes indicated that neural variability was quenched more when the arrow pointed left. Negative $t$ values in the left electrodes indicated that neural variability was quenched to a larger degree when the arrow pointed right. This demonstrated the contralateral lateralization of neural variability quenching in the discrimination experiment. $\boldsymbol{C}$, Same as in $\boldsymbol{A}$, but for the control experiment. $\boldsymbol{D}$, Same as in $\boldsymbol{B}$, but for the control experiment. Asterisks: significant differences across hemispheres (tw0-tailed $t$-test, $p<0.05$ ).

side of the visual field generated larger variability quenching in the neural responses of the contralateral hemisphere. To demonstrate this, we computed a lateralization index, which quantified the difference in magnitude of variability quenching when the cue pointed left versus right (see Materials and Methods). A negative value indicated that neural variability was quenched more when the arrow pointed right, and a positive value indicated that neural variability was quenched more when the arrow pointed left.

The lateralization index was negative in left electrodes and positive in right electrodes, indicating that variability quenching was larger in the contralateral electrodes to the attended visual field (Fig. 4A). There was a significant difference in the lateralization index across these two conditions (two-tailed $t$ test, $p=$ $0.01)$. Lateralized changes in neural variability were also apparent when examining the topography of lateralization indexes computed per electrode (Fig. 4B).

In contrast, computing the same lateralization index for responses in the control experiment demonstrated no contralateral lateralization of neural variability quenching (Fig. $4 C, D$ ). The lateralization index was positive for both right and left electrodes 

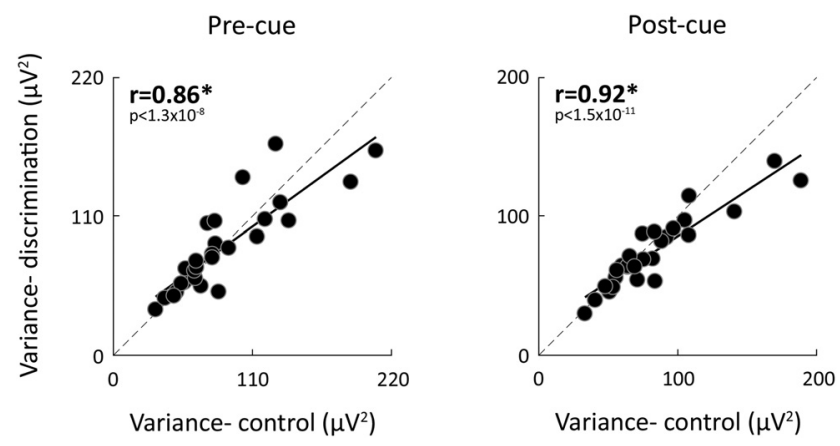

Figure 5. Differences in neural variability across subjects. Scatter plot presenting individual subjects' neural variability magnitudes before (left) and after (right) cue presentation in the discrimination versus control experiments (mean across cue types). Each point represents a single subject. Pearson's correlation coefficients are noted in each panel. Solid line indicates linear fit. Dotted line indicates unity line. ${ }^{*} p<0.05$, significant correlation (randomization test).

with larger positive values in the left electrodes (i.e., opposite effect to that described above). This demonstrated that the larger reduction in variability in the contralateral hemisphere to the attended visual field was apparent only when subjects were motivated to attend the cues (i.e., when the cues provided taskrelevant information) and not when the cues were meaningless.

\section{Between-subject differences in neural variability}

In agreement with previous studies from our laboratory (Arazi et al., 2017b), we found large differences in neural variability magnitudes across subjects, which were highly reproducible across experiments. Neural variability magnitudes in the precue interval (from $-200 \mathrm{~ms}$ to cue onset) and postcue interval were strongly, positively correlated across the two experiments for all three cue types $\left(r_{(27)}>0.86, p<0.001\right.$; Fig. 5$)$.

\section{Attentional benefits are associated with larger initial neural variability and larger neural variability quenching}

The behavioral benefit of allocating attention also varied considerably across subjects. We quantified the benefit of attention for each subject by computing the difference in accuracy between the valid and invalid conditions (i.e., "attentional effect"). Whereas some subjects exhibited an attentional effect as high as 10\%, others had an attentional effect that was close to zero (mean $=3.9 \%$, $\mathrm{SD}=2.6 \%$ ).

Individual attentional effects were positively correlated with the magnitudes of neural variability whether calculated in the precue or postcue period. Subjects with larger overall neural variability had larger attentional benefits when measuring neural variability in either left (postcue: $r_{(27)}=0.61$; precue: $r_{(27)}=0.54$; $p<0.003$; mean across cue types) or right (postcue: $r_{(27)}=0.53$; precue: $r_{(27)}=0.41 ; p<0.04$; mean across cue types) electrodes (Fig. 6). Assessing this relationship separately in each electrode and examining spatial topography of correlation values revealed that correlations were strongest in occipital-parietal electrodes located over the visual system (Fig. 6C).

The relationship described above was specific to the attentional benefit of individual subjects (i.e., the improvement in accuracy due to attention) and not associated with their absolute perceptual performance. That is, the tilt angle of the target stimulus, which was established via the staircase procedure, was not significantly correlated with the magnitude of neural variability in either left (precue: $r_{(27)}=-0.06$; postcue: $r_{(27)}=-0.13 ; p>$
0.52 ) or right (precue: $r_{(27)}=-0.11$; postcue: $r_{(27)}=-0.1 ; p>$ $0.58)$ electrodes.

Individual neural variability magnitudes were positively correlated with the difference in magnitude of variability quenching between the control and orientation discrimination experiments. Specifically, individuals with larger overall neural variability quenched more in the orientation discrimination experiment. This was demonstrated by positive, significant correlations in the left $\left(r_{(27)}=0.46 ; p=0.017\right.$; mean across cue types $)$ and right $\left(r_{(27)}=0.44 ; p=0.022\right.$; mean across cue types $)$ electrodes. This suggested that individual subjects with larger attentional effects have larger initial neural variability magnitudes, which were more strongly quenched when an alerting cue was presented.

Indeed, individuals with lower neural variability in the discrimination experiment relative to the control experiment exhibited larger attentional benefits (Fig. 7). This was apparent in significant positive correlations between the subjects' attentional benefits and the difference between their neural variability magnitudes in the control and discrimination experiments as quantified in left $\left(r_{(27)}>0.42, p<0.015\right)$ or right $\left(r_{(27)}>0.41, p<\right.$ $0.035)$ electrodes. These correlations suggest that individual subjects with larger reductions in neural variability, when alerted by the cues during the discrimination task, attained larger attentional benefits. These correlations were positive and strong in occipital-parietal electrodes (Fig. 7C), indicating that this relationship was specific to visual system areas.

This relationship was also specific to the attentional benefit of individual subjects (i.e., the improvement in accuracy due to attention) and not associated with their absolute perceptual performance. The tilt angle of the target stimulus was not significantly correlated with individual differences in the magnitude of variability across experiments, as quantified in the left $(-0.13<$ $\left.r_{(27)}<-0.06, p>0.52\right)$ or right $\left(-0.11<r_{(27)}<0, p>0.55\right)$ electrodes.

\section{Measurements of non-neural noise}

We performed two control analyses to exclude alternative interpretations of our data. First, we examined the stability of individual EEG recording as estimated by the electrode offset (an analogous measure to impedance in EEG systems with active electrodes). Electrode offsets differed across subjects but were below $\pm 10 \mathrm{mV}$ in all cases, which was far below the $\pm 40 \mathrm{mV}$ threshold recommended by the manufacturer for quality recordings. Individual differences in mean electrode offset across trials were not significantly correlated with individual neural variability magnitudes in the precue or postcue periods in the control $\left(-0.2<r_{(27)}<-0.18, p>0.29\right)$ or discrimination $(-0.25<$ $\left.r_{(27)}<-0.19, p>0.2\right)$ experiments. Similarly, the variability of electrode offset across trials was not significantly correlated with neural variability magnitudes in the precue or postcue periods in either the control $\left(-0.2<r_{(27)}<-0.15, p>0.3\right)$ or discrimination experiment $\left(-0.18<r_{(27)}<-0.17, p>0.38\right)$. These analyses showed that differences in the neural variability of individual subjects were not due to potential differences in the quality of their EEG recordings.

We also examined whether the amount of eye movements performed during each of the experiments had an impact on our estimates of neural variability given that eye movements alter the EEG signal (Yuval-Greenberg et al., 2008). On average, subjects fixated on the fixation spot equally well in both experiments. This was apparent when plotting the mean distance between gaze position and the fixation cross across trials and subjects for each experiment separately (Fig. 8A). We also compared gaze position 
A
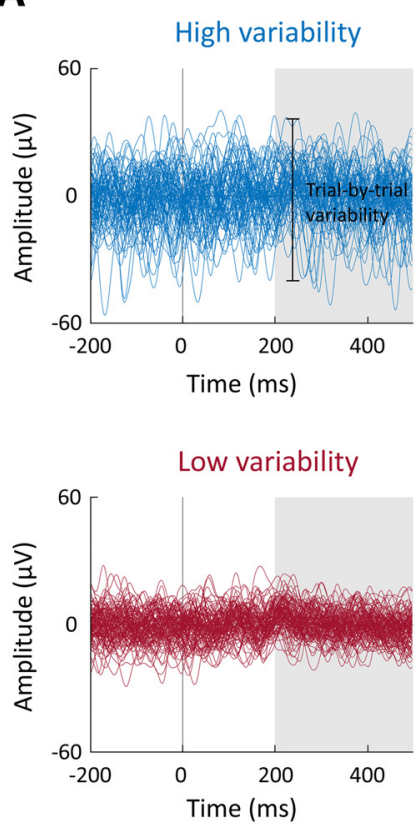

B
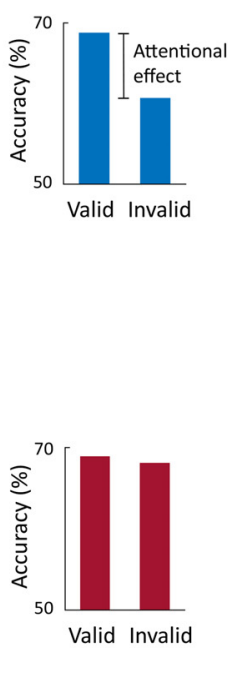

C
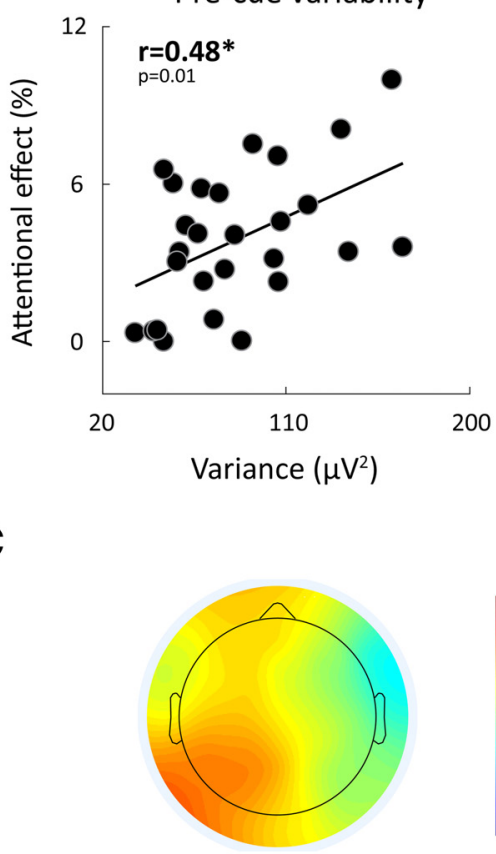

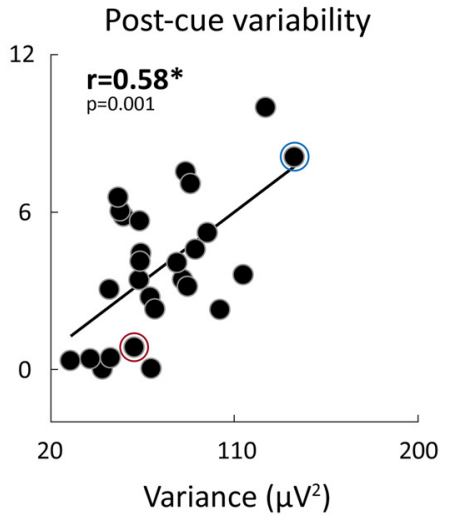

0.7
0.35
0

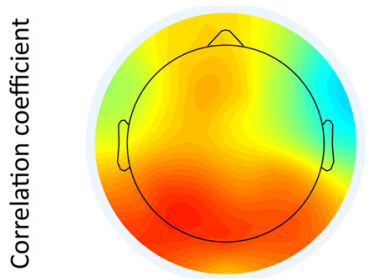

Figure 6. Greater attentional benefits were apparent in subjects with larger overall neural variability. $A$, Examples of the EEG traces from 2 individual subjects with relatively large (blue) or small (red) neural variability are presented next to their accuracy in the valid and invalid trials. $\boldsymbol{B}$, Scatter plots demonstrating the relationship between individual attentional effects and postcue or precue variability (mean across cue types and electrodes). Pearson's correlation coefficients are noted in each panel. ${ }^{*} p<0.05$, significant correlation (randomization test). Blue and red circles represent the 2 exemplary subjects. C, Topographic maps demonstrating the correlation coefficients between individual subjects' attentional effects and their magnitudes of neural variability in the precue (left) or postcue (right) period when calculated separately for each electrode.

A
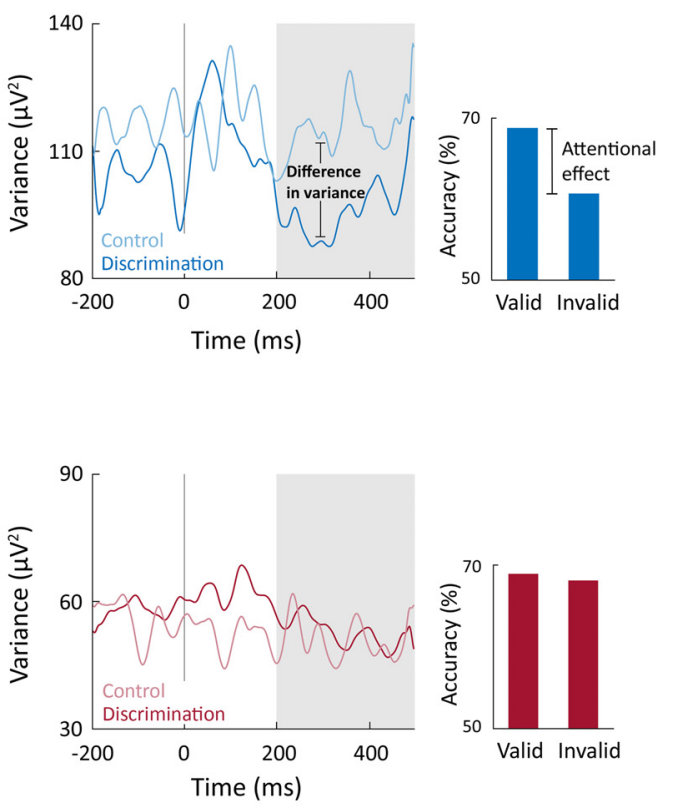

B

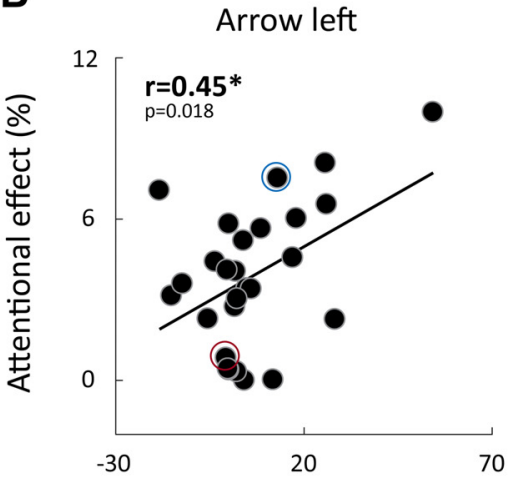

Arrow right

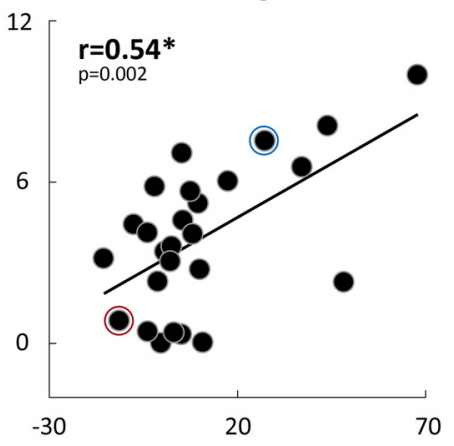

$-30$ 70

Difference in variance: control-discrimination $\left(\mu \mathrm{V}^{2}\right)$

C
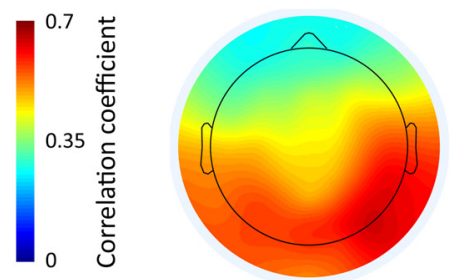

Figure 7. Larger attentional benefits are associated with larger differences in neural variability between control and discrimination experiments. $A$, Examples of trial-by-trial neural variability in the control and discrimination experiments from 2 subjects: one with a relatively large difference in neural variability across experiments (blue) and another with little difference across experiments (red). Attentional effects of the 2 subjects are also presented. $\boldsymbol{B}$, Scatter plots demonstrating the relationship between individual differences in neural variability between the control and discrimination experiments ( $250-500 \mathrm{~ms}$ after the cue, mean across right and left electrodes), and individual attentional effects. Pearson's correlation coefficients are noted in each panel. ${ }^{*} p<$ 0.05 , significant correlation (randomization test). Blue and red circles represent the 2 exemplary subjects. C, Topographic maps demonstrating the correlation coefficients between individual subjects' attentional effects and the difference in neural variability between control and discrimination experiments. Findings were similar when the cue pointed to the left (left panel) or to the right (right panel). 
A

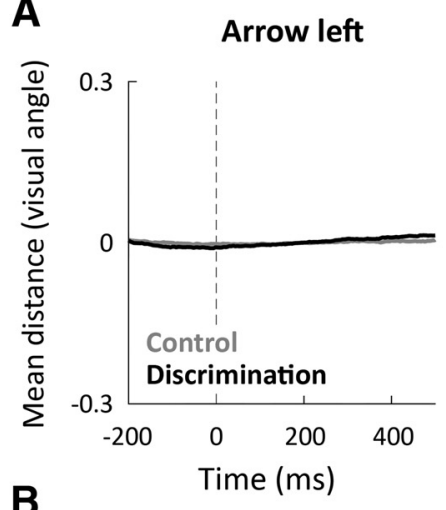

B
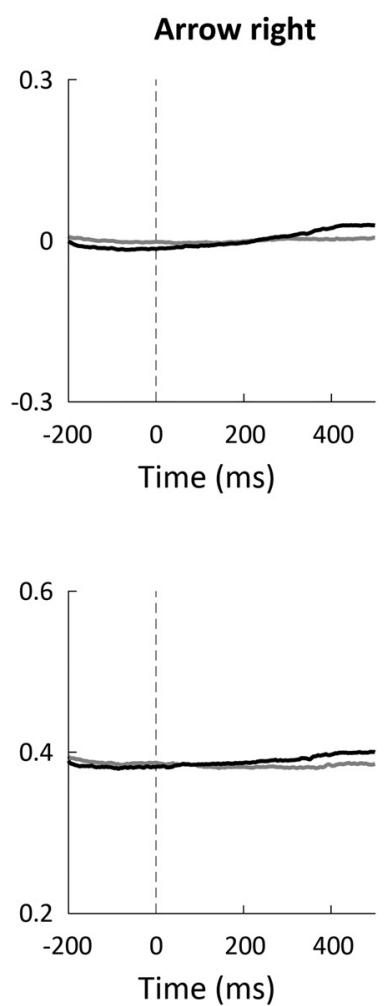

Double headed arrow
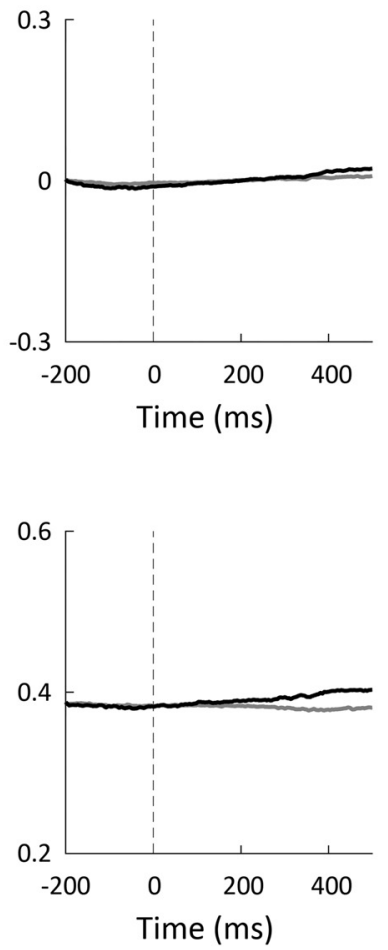

Figure 8. Eye movements did not differ across experiments, and gaze fixation was stable before/after cue presentation. $A$, The distance between gaze position and fixation spot (mean across trials and subjects) was stable before/after cue presentation and did not differ between the discrimination (black) and control (gray) experiments. $\boldsymbol{B}$, The SD across trials of the distance between gaze position and fixation cross was also stable before/after cue presentation and equivalent in both experiments. Each panel represents the results of a different cue type in units of visual angle. There were no significant differences across experiments in either measure (per time point, paired $t$ test, $p>0.1$ ).

variability across trials between the two experiments. We calculated the SD in gaze position across trials for each time point from -200 to $500 \mathrm{~ms}$ (Fig. $8 B$ ). Both measures were not significantly different across the control and discrimination experiments (paired-sample $t$ test for each of the time points, $p>0.11$ ). Furthermore, the gaze position variability of individual subjects was not correlated with their neural variability magnitudes in either the control $\left(-0.28<r_{(27)}<-0.24, p>0.15\right)$ or discrimination experiment $\left(0<r_{(27)}<0.21, p>0.29\right)$. This demonstrated that potential EEG artifacts caused by eye movements did not account for the reported results.

\section{Discussion}

It is well established that trial-by-trial neural variability is reduced ("quenched") by the presentation of visual stimuli (Churchland et al., 2010). Our results reveal that modulating attention through alertness or spatial attention further quenches neural variability. First, presenting cues that alerted the subjects to an upcoming task-relevant stimulus generated significantly larger neural variability quenching than identical cues presented in a passive control experiment without a task. These differences in neural variability across experiments were specific to EEG electrodes covering visual cortices (Figs. 2, 3), thereby demonstrating that alertness quenched neural variability specifically in the visual system. A second finding was that modulating spatial attention to the right or left side of the visual field generated a significantly larger decrease in neural variability in the contralateral visual areas of the attended visual field (Fig. 4). This suggests that allocating spatial attention decreases the variability in the cortical neural populations with receptive fields in the attended location.
While alertness and spatial attention are distinct components of attention that are likely governed by different brain networks (Petersen and Posner, 2012), we suggest that a common outcome of attention modulation is the decrease of neural variability in task-relevant neural populations.

Moreover, neural variability magnitudes of individual subjects were associated with their behavioral benefits from attention. Subjects with larger overall neural variability, who quenched their neural variability to a larger extent in the discrimination experiment, relative to the control experiment, enjoyed greater attentional benefits (Figs. 6, 7). Hence, the large differences across subjects in attentional effects may be partially explained by stable individual differences in overall neural variability (Fig. 5) and the ability to quench this variability with attentional mechanisms.

\section{Different components of attention}

Attention can be subdivided into three functional components: alerting, orienting, and executive control. Alerting is defined as achieving and maintaining an alert state; orienting is the selection of information from a specific location; and executive control is operating to resolve conflicts among responses (Petersen and Posner, 2012).

Here, we focused on the effects of alertness and spatial orienting (i.e., spatial attention). Alertness was examined by comparing neural variability across the discrimination and control experiments. While both experiments contained identical cues (arrows pointing to the right, left, or both directions), in the discrimination experiment, these cues were meaningful because they alerted the subject to the upcoming appearance of a task-relevant visual 
stimulus. In contrast, in the control experiment, there was no task and, therefore, no motivation to attend the cues. Our results demonstrate that, when the cues alerted subjects to upcoming stimuli, they generated a significant decrease in neural variability (Figs. 2, 3) and that the magnitude of this decrease in neural variability was associated with the attentional benefit of individual subjects (Fig. 7).

We also examined spatial orienting by shifting subjects' attention to the left or right visual field. These attentional shifts generated larger variability quenching in the contralateral hemisphere to the cued direction, demonstrating the spatial selectivity of changes in neural variability (Fig. 4). Neural variability changes associated with spatial attention were weaker than those associated with alertness.

\section{Attention, signal detection theory, and neural variability}

Our results resonate well with the principles of signal detection theory (Green and Swets, 1966), which suggest that perceptual capabilities are dependent on the signal-to-noise ratio of the neural response associated with the stimulus. This can be achieved by amplifying the relevant neural activity (i.e., increasing the signal) and/or by reducing the variability of the relevant neural activity (i.e., reducing the noise).

Numerous studies have demonstrated that attentional benefits are associated with an increase in the relevant neural activity. This was demonstrated by a variety of electrophysiology studies that reported increased firing rates of neurons encoding the attended stimulus in multiple areas of visual cortex (Mitchell et al., 2007; McAlonan et al., 2008; Buffalo et al., 2010; Davidesco et al., 2013; Thiele et al., 2016) and in PFC (Everling et al., 2002). Neuroimaging studies in humans have reported analogous increases in BOLD (Gandhi et al., 1999; Ress et al., 2000) and event-related potential (Hillyard and Anllo-Vento, 1998) responses in similar cortical areas. Several complementary studies have reported that allocating spatial attention reduces the variability of neural activity across trials as measured by the Fano factor of individual neurons (Mitchell et al., 2007; Thiele et al., 2016). Furthermore, the correlated variability evident in the responses of pairs of neurons was also reduced by allocating attention (Cohen and Maunsell, 2009; Mitchell et al., 2009; Rabinowitz et al., 2015).

Our results reveal, for the first time, that spatial orienting also quenches neural variability in humans, and demonstrate that variability quenching is lateralized to the contralateral hemisphere of the attended visual field (i.e., stronger in the cortical areas that process the attended stimulus). The results extend findings from the only study to date that has examined the relationship between alertness and response variability in humans. This study reported that fMRI responses were less variable across trials when subjects performed a target detection task at fixation compared with passively observing the same stimulus (Broday-Dvir et al., 2018). Using EEG, we demonstrate, for the first time, that the reduction in neural variability was specific to the postcue period (i.e., 250-500 ms after cue presentation), when subjects were actively anticipating the presentation of the target stimulus. Furthermore, our results are the first to demonstrate that individual magnitudes of variability quenching explain individual differences in behavioral performance.

\section{Individual differences in neural variability}

A variety of recent studies have shown that neural variability measures are informative regarding the cognitive and perceptual abilities of individual subjects as well as their clinical state (Dinstein et al., 2015). For example, it has been reported that individuals with larger ongoing neural variability, as measured by the amplitude of fMRI fluctuations, exhibit better performance on several basic cognitive measures (Garrett et al., 2011, 2013, 2015). It has been hypothesized that larger neural variability is an indicator of larger neural complexity, which may be beneficial for cognitive flexibility and the exploration of multiple solutions (McIntosh et al., 2008).

In parallel, others have reported that individuals with better contrast-discrimination thresholds exhibit larger neural variability quenching (Arazi et al., 2017a). It has also been shown that perception of weak stimuli is better on trials with lower neural variability (Schurger et al., 2010, 2015). Interestingly, unconscious patients exhibit more stable (i.e., less variable) ongoing neural activity but do not exhibit an increase in neural stability after stimulus presentation as found in controls (Schurger et al., 2015). Together, current evidence suggests that individuals with larger ongoing neural variability, who quench neural variability to a larger extent after stimulus presentation, exhibit superior behavioral performance. Our results further extend these findings in demonstrating that individuals with larger overall neural variability and larger reductions of neural variability following informative cues benefit more from allocating attention.

\section{Flexibility of neural variability}

When considering the potential relationships between neural variability and behavior, it is important to note that individual differences in neural variability are remarkably stable over time in adults (Arazi et al., 2017b). While neural variability is much larger during early periods of development that involve intense learning and plasticity (Olveczky et al., 2005), it seems to solidify into a stable individual characteristic by adulthood. These stable individual differences in neural variability were also apparent in the current study (Fig. 5). This suggests that the flexible modulation of neural variability by mechanisms of attention is limited by stable intrinsic mechanisms that govern/constrain the magnitude of neural variability in individual subjects.

\section{Alpha power suppression and attention}

Previous EEG and MEG studies have revealed that spatial attention suppresses the fluctuation of alpha-band activity following cue presentation, during the time window where subjects are expecting a visual stimulus to appear at the cued location (Thut et al., 2006; Siegel et al., 2008; Ikkai et al., 2016). Alpha suppression was found in occipital and parietal electrodes/sensors, contralateral to the attended position, presumably reflecting a higher level of cortical activation that may facilitate the processing of the stimulus (Pfurtscheller and Lopes da Silva, 1999; Klimesch, 2012). Neural variability quenching is strongly correlated with the suppression of broadband neural oscillations, including alpha-band suppression (Arazi et al., 2017a). This suggests that neural variability quenching and alpha-band suppression are associated measures of a mutual phenomenon, whereby allocation of attention leads to an increase in firing rates of task-relevant neural populations, decrease in lower frequency ongoing neural oscillations, and reduced trial-by-trial neural variability, all of which contribute to an increase in signal-to-noise ratio. 


\section{Conclusion}

It has been proposed that allocating attention to a stimulus leads to an increase in the signal-to-noise ratio of the neural populations encoding the attended stimulus. The current study extends the existing literature by demonstrating that manipulating alertness and spatial attention generates a decrease in trail-by-trial neural variability/noise of relevant visual neural populations in humans. While these components of attention may be governed by different brain systems (Petersen and Posner, 2012), both components seem to generate a reduction in neural variability. Most importantly, individual magnitudes of variability quenching partially explained individual differences in attention benefits. While neural variability is a remarkably stable individual characteristic in adulthood, these results demonstrate that mechanisms of attention control neural variability in a flexible and behaviorally relevant manner.

\section{References}

Arazi A, Censor N, Dinstein I (2017a) Neural variability quenching predicts individual perceptual abilities. J Neurosci 37:97-109.

Arazi A, Gonen-Yaacovi G, Dinstein I (2017b) The magnitude of trial-bytrial neural variability is reproducible over time and across tasks in humans. eNeuro 4:ENEURO.0292-17.2017.

Arieli A, Sterkin A, Grinvald A, Aertsen A (1996) Dynamics of ongoing activity: explanation of the large variability in evoked cortical responses. Science 273:1868-1871.

Brainard DH (1997) The psychophysics toolbox. Spat Vis 10:433-436.

Broday-Dvir R, Grossman S, Furman-Haran E, Malach R (2018) Quenching of spontaneous fluctuations by attention in human visual cortex. Neuroimage 171:84-98.

Bromberg-Martin ES, Matsumoto M, Hikosaka O (2010a) Distinct tonic and phasic anticipatory activity in lateral habenula and dopamine neurons. Neuron 67:144-155.

Bromberg-Martin ES, Matsumoto M, Hikosaka O (2010b) Dopamine in motivational control: rewarding, aversive, and alerting. Neuron 68:815834.

Buffalo EA, Fries P, Landman R, Liang H, Desimone R (2010) A backward progression of attentional effects in the ventral stream. Proc Natl Acad Sci U S A 107:361-365.

Carandini M (2004) Amplification of trial-to-trial response variability by neurons in visual cortex. PLoS Biol 2:e264.

Carrasco M (2011) Visual attention: the past 25 years. Vision Res 51:14841525.

Churchland MM, Yu BM, Cunningham JP, Sugrue LP, Cohen MR, Corrado GS, Newsome WT, Clark AM, Hosseini P, Scott BB, Bradley DC, Smith MA, Kohn A, Movshon JA, Armstrong KM, Moore T, Chang SW, Snyder LH, Lisberger SG, Priebe NJ, et al. (2010) Stimulus onset quenches neural variability: a widespread cortical phenomenon. Nat Neurosci 13:369-378.

Cohen MR, Maunsell JH (2009) Attention improves performance primarily by reducing interneuronal correlations. Nat Neurosci 12:1594-1600.

Davidesco I, Harel M, Ramot M, Kramer U, Kipervasser S, Andelman F, Neufeld MY, Goelman G, Fried I, Malach R (2013) Spatial and object-based attention modulates broadband high-frequency responses across the human visual cortical hierarchy. J Neurosci 33: $1228-1240$

Delorme A, Makeig S (2004) EEGLAB: an open source toolbox for analysis of single-trial EEG dynamics including independent component analysis. J Neurosci Methods 134:9-21.

Dinstein I, Heeger DJ, Behrmann M (2015) Neural variability: friend or foe? Trends Cogn Sci 19:322-328.

Everling S, Tinsley CJ, Gaffan D, Duncan J (2002) Filtering of neural signals by focused attention in the monkey prefrontal cortex. Nat Neurosci 5:671-676.

Fan J, McCandliss BD, Sommer T, Raz A, Posner MI (2002) Testing the efficiency and independence of attentional networks. J Cogn Neurosci 14:340-347.

Fan J, McCandliss TB, Fossella J, Flombaum JI, Posner MI (2005) The activation of attentional networks. Neuroimage 26:471-479.

Fan J, Gu X, Guise KG, Liu X, Fossella J, Wang H, Posner MI (2009) Testing the behavioral interaction and integration of attentional networks. Brain Cogn 70:209-220.

Finn IM, Priebe NJ, Ferster D (2007) The emergence of contrastinvariant orientation tuning in simple cells of cat visual cortex. Neuron 54:137-152.

Gandhi SP, Heeger DJ, Boynton GM (1999) Spatial attention affects brain activity in human primary visual cortex. Proc Natl Acad Sci U S A 96:3314-3319.

Garrett DD, Kovacevic N, McIntosh AR, Grady CL (2011) The importance of being variable. J Neurosci 31:4496-4503.

Garrett DD, Kovacevic N, McIntosh AR, Grady CL (2013) The modulation of BOLD variability between cognitive states varies by age and processing speed. Cereb Cortex 23:684-693.

Garrett DD, Nagel IE, Preuschhof C, Burzynska AZ, Marchner J, Wiegert S, Jungehülsing GJ, Nyberg L, Villringer A, Li SC, Heekeren HR, Bäckman L, Lindenberger U (2015) Amphetamine modulates brain signal variability and working memory in younger and older adults. Proc Natl Acad Sci U S A 112:7593-7598.

Goris RL, Movshon JA, Simoncelli EP (2014) Partitioning neuronal variability. Nat Neurosci 17:858-865.

Green DM, Swets JA (1966) Signal detection theory and psychophysics. New York: John Wiley.

He BJ (2013) Spontaneous and task-evoked brain activity negatively interact. J Neurosci 33:4672-4682.

Hillyard SA, Anllo-Vento L (1998) Event-related brain potentials in the study of visual selective attention. Proc Natl Acad Sci U S A 95:781787.

Hussar C, Pasternak T (2010) Trial-to-trial variability of the prefrontal neurons reveals the nature of their engagement in a motion discrimination task. Proc Natl Acad Sci U S A 107:21842-21847.

Ikkai A, Dandekar S, Curtis CE (2016) Lateralization in alpha-band oscillations predicts the locus and spatial distribution of attention. PLoS One 11:e0154796.

Kappenman ES, Luck SJ (2010) The effects of electrode impedance on data quality and statistical significance in ERP recordings. Psychophysiology 47:888-904.

Kastner S, Pinsk MA, De Weerd P, Desimone R, Ungerleider LG (1999) Increased activity in human visual cortex during directed attention in the absence of visual stimulation. Neuron 22:751-761.

Klimesch W (2012) Alpha-band oscillations, attention, and controlled access to stored information. Trends Cogn Sci 16:606-617.

McAlonan K, Cavanaugh J, Wurtz RH (2008) Guarding the gateway to cortex with attention in visual thalamus. Nature 456:391-394.

McIntosh AR, Kovacevic N, Itier RJ (2008) Increased brain signal variability accompanies lower behavioral variability in development. PLoS Comput Biol 4:e1000106.

Mitchell JF, Sundberg KA, Reynolds JH (2007) Differential attentiondependent response modulation across cell classes in macaque visual area V4. Neuron 55:131-141.

Mitchell JF, Sundberg KA, Reynolds JH (2009) Spatial attention decorrelates intrinsic activity fluctuations in macaque area V4. Neuron 63:879888.

Monier C, Chavane F, Baudot P, Graham LJ, Frégnac Y (2003) Orientation and direction selectivity of synaptic inputs in visual cortical neurons: a diversity of combinations produces spike tuning. Neuron 37: $663-680$.

Moore T, Zirnsak M (2017) Neural mechanisms of selective visual attention. Annu Rev Psychol 68:47-72.

Noudoost B, Chang MH, Steinmetz NA, Moore T (2010) Top-down control of visual attention. Curr Opin Neurobiol 20:183-190.

Olveczky BP, Andalman AS, Fee MS (2005) Vocal experimentation in the juvenile songbird requires a basal ganglia circuit. PLoS Biol 3:e153.

Petersen SE, Posner MI (2012) The attention system of the human brain: 20 years after. Annu Rev Neurosci 35:73-89.

Pfurtscheller G, Lopes da Silva FH (1999) Event-related EEG/MEG synchronization and desynchronization: basic principles. Clin Neurophysiol 110:1842-1857.

Posner MI (1980) Orienting of attention. Q J Exp Psychol 32:3-25.

Rabinowitz NC, Goris RL, Cohen M, Simoncelli EP (2015) Attention stabilizes the shared gain of V4 populations. Elife 4:1-24.

Ress D, Backus BT, Heeger DJ (2000) Activity in primary visual cortex 
predicts performance in a visual detection task. Nat Neurosci 3:940-945.

Saproo S, Serences JT (2010) Spatial attention improves the quality of population codes in human visual cortex. J Neurophysiol 104: 885-895.

Schurger A, Pereira F, Treisman A, Cohen JD (2010) Reproducibility distinguishes conscious from nonconscious neural representations. Science 327:97-99.

Schurger A, Sarigiannidis I, Naccache L, Sitt JD, Dehaene S (2015) Cortical activity is more stable when sensory stimuli are consciously perceived. Proc Natl Acad Sci U S A 112:E2083-E2092.

Siegel M, Donner TH, Oostenveld R, Fries P, Engel AK (2008) Neuronal synchronization along the dorsal visual pathway reflects the focus of spatial attention. Neuron 60:709-719.

Silver MA, Ress D, Heeger DJ (2007) Neural correlates of sustained spatial attention in human early visual cortex. J Neurophysiol 97:229-237.
Thiele A, Brandt C, Dasilva M, Gotthardt S, Chicharro D, Panzeri S, Distler C (2016) Attention induced gain stabilization in broad and narrow-spiking cells in the frontal eye-field of macaque monkeys. J Neurosci 36: 7601-7612.

Thut G, Nietzel A, Brandt SA, Pascual-Leone A (2006) Alpha band electroencephalographic activity over occipital cortex indexes visuospatial attention bias and predicts visual target detection. J Neurosci 26:9494-9502.

Xuan B, Mackie MA, Spagna A, Wu T, Tian Y, Hof PR, Fan J (2016) The activation of interactive attentional networks. Neuroimage 129: $308-319$.

Yeshurun Y, Carrasco M (1998) Attention improves or impairs visual performance by enhancing spatial resolution. Nature 396:72-75.

Yuval-Greenberg S, Tomer O, Keren AS, Nelken I, Deouell LY (2008) Transient induced gamma-band response in EEG as a manifestation of miniature saccades. Neuron 58:429-441. 